\title{
Accurate measurement of uncorrelated energy spread in electron beam
}

\author{
Sergey Tomin, ${ }^{*}$ Igor Zagorodnov®, Winfried Decking, Nina Golubeva, and Matthias Scholz \\ Deutsches Elektronen-Synchrotron, Notkestrasse 85, 22607 Hamburg, Germany
}

(Received 19 March 2021; accepted 19 May 2021; published 2 June 2021)

\begin{abstract}
We present measurements of slice energy spread at the injector section of the European X-Ray Free Electron Laser for an electron bunch with charge of $250 \mathrm{pC}$. Two methods considered in the paper are based on measurements at the dispersive section after a transverse deflecting structure (TDS). The first approach uses measurements at different beam energies. We show that with a proper scaling of the TDS voltage with the beam energy the rms error of the measurement is less than $0.3 \mathrm{keV}$ for the energy spread of $6 \mathrm{keV}$. In the second approach we demonstrate that keeping the beam energy constant but adjusting only the optics we are able to simplify the measurement complexity and to reduce the rms error below $0.1 \mathrm{keV}$. The accuracy of the measurement is confirmed by numerical modeling including beam transport effects and collective beam dynamics of the electron beam. The slice energy spread measured at the European XFEL for the beam charge of $250 \mathrm{pC}$ is nearly 3 times lower as the one reported recently at SwissFEL for the same cathode material and the beam charge of $200 \mathrm{pC}$.
\end{abstract}

DOI: $10.1103 /$ PhysRevAccelBeams.24.064201

\section{INTRODUCTION}

The small emittance and the low energy spread of the electron beam required at X-Ray Free Electron Lasers (XFELs) can cause the microbunching instability [1,2] and destroy the lasing. On the other hand, a large initial energy spread will hinder a proper compression of the bunch and will lead to intolerable energy spread after compression. Currently, the modeling of the electron beam dynamics does not correctly reproduce the slice energy spread measurement, e.g., see [3,4]. Therefore, a reliable, highresolution method to measure the uncorrelated (or slice) energy spread is crucial for the optimal operation of the modern facilities.

In order to measure the slice energy spread a standard approach with a transverse deflector and the dispersive section is used. However, it shows only a low resolution (of several $\mathrm{keV}$ ) due to impact of OTR screen resolution, the betatron beam size and the deflector strength on the measurement. The energy spread induced by deflector can be excluded with a set of measurements with different deflector amplitudes. Such experiments have been done at PITZ [5].

Recently in [4] it was suggested to carry out the measurements at different electron beam energies. For

\footnotetext{
*Corresponding author. sergey.tomin@desy.de

Published by the American Physical Society under the terms of the Creative Commons Attribution 4.0 International license. Further distribution of this work must maintain attribution to the author(s) and the published article's title, journal citation, and DOI.
}

the setup used at SwissFEL-deflector at constant energy and an acceleration section after it-the authors have written a polynomial equation of the second order and analyzed the accuracy of the coefficient reconstruction relative to statistical and systematic errors. On the basis of this analysis they concluded that the accuracy of the measurements for their setup could be better than $1 \mathrm{keV}$. In order to exclude the impact of the deflector the authors in [4] suggested to carry out additional sets of measurements, but it was not done and an analytical estimation was used instead.

The energy scan method seems to be simple. However at the layout of the European XFEL this method requires modifications and even with the changes described in this paper it has not shown the expected performance.

At this paper we present the measurement at the European XFEL with layout shown in Fig. 1. The situation is different as compared to SwissFEL case. The deflector is placed after the acceleration section, and the beam energy in it changes during the experiment. This case was mentioned in [4] too and it was suggested to use a polynomial equation of the third order with additional sets of measurements in order to exclude the impact of the deflector.

If we scan energy then with our setup the accuracy of the reconstruction of the third order polynomial is low and very sensitive to errors. In order to overcome this we suggest a scaling of the deflector voltage with the beam energy. First, it allows one to reduce the order of the polynomial equation; second, one can avoid needing to take additional sets of measurement with different deflector amplitudes. Finally, and most importantly, the suggested method is considerably 


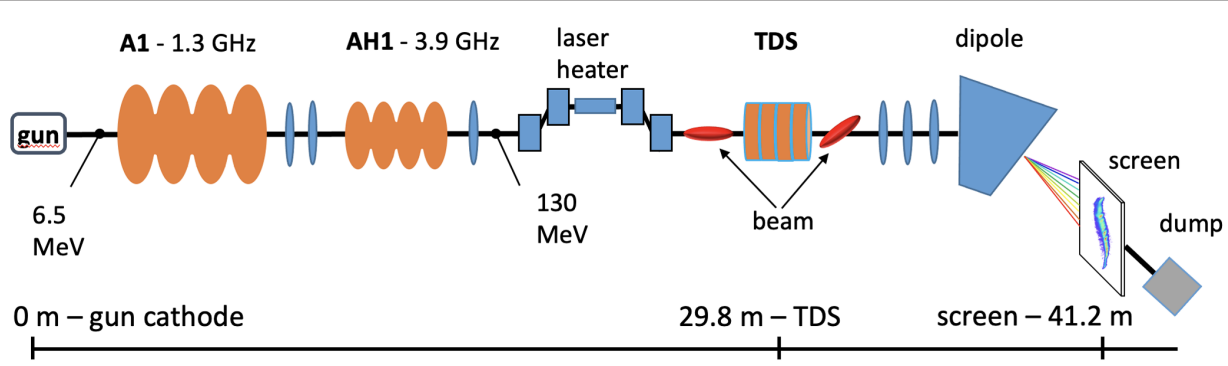

FIG. 1. The setup of the experiment at the injector section of the European XFEL.

more accurate in reconstructing the coefficients, and results in more accurate energy spread measurements.

The experiments described here are performed at the EuXFEL injector where the beam energy is relatively low. In order to analyze the accuracy of the measurement we have done numerical modeling including beam transport effects and collective beam dynamics of the electron beam. We have found that the radio-frequency (rf) focusing impacts the beam considerably and a matching the beam to the optics before the TDS for each energy is necessary. Additionally we see in the modeling and in the experiment that due to collective effects the emittance and the energy spread are not constant during the energy scan.

Applying the method based on the energy scan in practice we have found that, for our setup and a small interval of energy change available, the method is very time-intensive due to the beam matching and the optics scaling. In the measurements we have failed to obtain reliable data which would allow an accurate reconstruction. Hence we developed another method and have used the data from the energy scan experiments only to show the consistence with the results obtained by the second method.

In the second approach we keep the beam energy constant and avoid time-consuming matching of the beam before the TDS. By adjusting the strength of the quadrupoles after the TDS we are able to carry out independent scans in dispersion, in TDS strength and in beta-function on the OTR screen. The method is fast and allows one to obtain accurate measurements of slice energy spread with a resolution better than $0.1 \mathrm{keV}$.

The paper is organized as follows, first the measurement methods and their analysis are described in Sec. II. Second, the beam dynamics modeling of the approaches is considered in Sec. III. Finally, in Sec. IV, the results of the measurements at the European XFEL injector and their analysis are presented.

\section{METHODS OF THE MEASUREMENT AND THEIR ANALYSIS}

For the setup of Fig. 1 the measured beam size $\sigma_{M}$ on the screen can be written as

$$
\begin{aligned}
& \sigma_{M}^{2}=\sigma_{R}^{2}+\frac{E_{0}}{E} \sigma_{B}^{2}+\frac{D^{2}}{E^{2}} \sigma_{E}^{2}+\frac{(D e k V)^{2} E_{0}}{E^{3}} \sigma_{I}^{2}, \\
& \sigma_{B}^{2}=\frac{\beta_{x} \epsilon_{n}}{\gamma_{0}}, \quad \sigma_{I}^{2}=\frac{\epsilon_{n}\left(\beta_{y}^{0}+0.25 L^{2} \gamma_{y}^{0}-L \alpha_{y}^{0}\right)}{\gamma_{0}},
\end{aligned}
$$

where $E$ is the beam energy, $E_{0}$ is the reference beam energy, $\sigma_{R}$ is the screen resolution, $\beta_{x}$ is the optical function at the position of the screen, $\epsilon_{n}$ is the normalized beam emittance, $\gamma_{0}=\frac{E_{0}}{m_{e} c^{2}}$ is the relative beam energy, $D$ is the horizontal dispersion at the screen position; $\beta_{y}^{0}, \gamma_{y}^{0}$ and $\alpha_{y}^{0}$ are the Twiss parameters at the beginning of the TDS; $k, V$, and $L$ are the wave number, voltage and length of the TDS, respectively, and $e$ is the electron charge.

In this section we propose and validate two methods to measure the slice energy spread. The parameters used in the simulations are listed in Table I. These parameters are close to estimations obtained in the experiment. The resolution of the OTR screen is $28 \mu \mathrm{m}$ and it agrees with other publications: $10-20 \mu \mathrm{m}$ in [6], $30 \mu \mathrm{m}$ in [4].

\section{A. Method based on energy scan}

If we neglect the contribution of the screen resolution, the betatron size and the TDS effect to the beam size on the screen $\sigma_{M}$, see Eq. (1), then the energy spread can be estimated as

TABLE I. Simulation parameters.

\begin{tabular}{lcc}
\hline \hline Parameter & Units & Value \\
\hline OTR resolution, $\sigma_{R}$ & $\mu \mathrm{m}$ & 28 \\
Normalized emittance, $\epsilon_{n}$ & $\mu \mathrm{m}$ & 0.4 \\
Reference optical $\beta$-function at OTR, $\beta_{x}^{0}$ & $\mathrm{~m}$ & 0.6 \\
Reference dispersion, $D_{0}$ & $\mathrm{~m}$ & 1.2 \\
Optical $\beta$-function at TDS, $\beta_{y}^{0}$ & $\mathrm{~m}$ & 4.3 \\
Optical $\alpha$-function at TDS, $\alpha_{y}^{0}$ & & 1.9 \\
Wave number of TDS, $k$ & $1 / \mathrm{m}$ & 58.7 \\
Length of TDS, $L$ & $\mathrm{~m}$ & 0.7 \\
Reference voltage of TDS, $V_{0}$ & $\mathrm{MV}$ & 0.61 \\
Reference energy, $E_{0}$ & $\mathrm{MeV}$ & 130 \\
\hline \hline
\end{tabular}




$$
\sigma_{E} \approx \frac{E}{D} \sigma_{M}
$$

The error of this estimation is defined by the resolution

$$
R_{\sigma_{E}}=\frac{E}{D} \sqrt{\sigma_{R}^{2}+\frac{E_{0}}{E} \sigma_{B}^{2}+\frac{(D e k V)^{2} E_{0}}{E^{3}} \sigma_{I}^{2}} .
$$

In order to increase the resolution it was suggested in [4] to "perform beam size measurements for different energies and deflector voltages and to fit the data" with Eq. (1). Note that Eq. (1) uses a more accurate approximation of the last term compared to [4] where the authors used $\sigma_{I}^{2}=\epsilon_{n} \beta_{y}^{0} / \gamma_{0}$.

If we keep the voltage of the deflector constant and change only the beam energies then we can fit the measurements to Eq. (1) in hope to reconstruct all coefficients of this polynomial. We simulated with Eq. (1) a measurement of the beam size $\sigma_{M}$ with constant TDS voltage $V_{0}$ and the beam energy changing between 90 and $190 \mathrm{MeV}$ with step of $10 \mathrm{MeV}$. At each beam energy we simulate 30 measurements of the beam size $\sigma_{M}$ with random error of $2 \%$. We consider the slice energy spread between 0.5 and $7 \mathrm{keV}$. In the fit we used the simplex search method of Lagarias et al. [7].

From numerical experiment we have found that the rms error of the reconstruction of energy spread is larger than $2 \mathrm{keV}$. Under the rms error of reconstruction in the paper we mean the value defined as

$$
\Delta_{\sigma_{E}}=\sqrt{\frac{1}{N} \sum_{i=1}^{N}\left(\sigma_{E}-\sigma_{E}^{0}\right)^{2}},
$$

where $N$ is the number of shots (reconstructions), $\sigma_{E}$ is the energy spread obtained from the reconstruction (of the polynomial coefficient from the simulated measurements) and $\sigma_{E}^{0}$ is true energy spread used in the simulation of the reconstruction procedure. In order to estimate this error we used 100 shots at each energy spread point.

In order to reduce the error we can do an additional scan with different deflector voltages to estimate the last term in Eq. (1). With this estimation we reduce the error of the reconstruction. However, we will not analyze this approach here and suggest below another technique to reduce the order of the polynomial and to increase the accuracy of the reconstruction of the polynomial coefficients. It can be achieved if we will keep constant not the deflector voltage $V$ but the streaking parameter:

$$
S_{0}=\sqrt{\beta_{y} \beta_{y}^{0}} \sin \left(\Delta \mu_{y}\right) K_{0}, \quad K_{0}=\frac{e V_{0} k}{E_{0}}
$$

where $\Delta \mu_{y}$ is the phase advance between the middle of the TDS and the OTR screen, $\beta_{y}^{0}$ is the optical function at the TDS, $\beta_{y}$ is the optical function at the position of the OTR and the voltage $V_{0}$ is a fixed value which produces the desired streak $S_{0}$ at the fixed beam energy $E_{0}$.

In the following we adjust the voltage of the TDS proportionally to the beam energy:

$$
V(E)=\frac{V_{0}}{E_{0}} E .
$$

If we put Eq. (6) in Eq. (1) then we reduce the order of the polynomial from the third to the second one:

$$
\sigma_{M}^{2}=\sigma_{R}^{2}+\frac{E_{0}}{E} \sigma_{B I}^{2}+\frac{D^{2}}{E^{2}} \sigma_{E}^{2}, \quad \sigma_{B I}^{2}=\sigma_{B}^{2}+\left(D K_{0} \sigma_{I}\right)^{2} .
$$

We simulated with Eq. (7) a measurement of the beam size $\sigma_{M}$ with the beam energy changing between 90 and $190 \mathrm{MeV}$ with step of $10 \mathrm{MeV}$. We used the same errors and the reconstruction algorithm as in the previous example. The results of the reconstruction are shown in Fig. 2 and the error of the reconstruction of energy spread is nearly $0.3 \mathrm{keV}$ at the energy spread of $6 \mathrm{keV}$.

However, in both the experiment and the beam dynamics simulations we have not been able to show this accuracy. In Sec. III, we will show with beam dynamics simulations that in order to use this method we have to match the beam to the optics at each beam energy. It requires considerable effort and hard to achieve and thus the accuracy of the measurements suffer. Additionally, in the modeling and the experiment we have seen that the slice emittance is not constant.
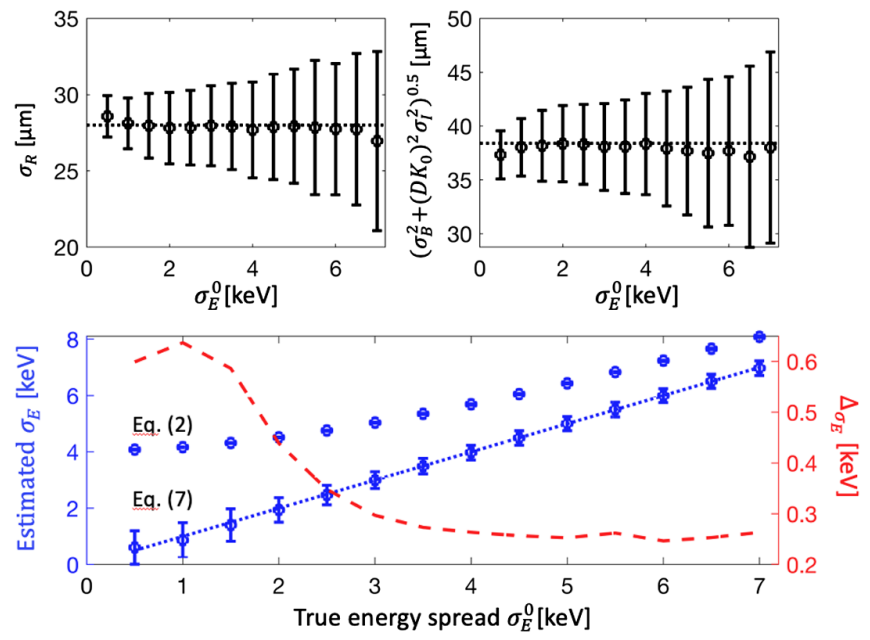

FIG. 2. Performance of the method based on the energy scan. Circles shows reconstructed mean values. Error bars give the standard deviation error. The red dashed curve presents the rms error of the reconstruction. Dotted blue curves show true values used for the simulation. 


\section{B. Method based on dispersion scan}

In this section we present another method which use constant beam energy $E_{0}$ and avoids the above described difficulties. The method shows much better resolution theoretically and it is easy to use experimentally.

We have developed a special optics described in the next section. Using only a few quadrupoles between the TDS and the OTR screen we are able to change the dispersion $D$ at the OTR position keeping $\beta_{x}$-function constant with only moderate changes in $\beta_{y}$-function and in the streaking $S$.

We start with changing of the TDS voltage $V$ and fit the measured slice size $\sigma_{M}$ to the quadratic polynomial:

$$
\sigma_{M}^{2}=A_{V}+B_{V} V^{2}
$$

During the scan we keep the dispersion at constant value $D_{0}$.

At the second step we keep constant the TDS voltage at $V_{0}$ and change the dispersion $D$. We fit the measured slice size $\sigma_{M}$ to the quadratic polynomial:

$$
\sigma_{M}^{2}=A_{D}+B_{D} D^{2}
$$

After these two fits we are able to find out all terms of Eq. (1):

$$
\begin{aligned}
& \sigma_{E}=\frac{E_{0}}{D_{0}} \sqrt{A_{D}-A_{V}}, \quad \sigma_{I}=\frac{E_{0}}{D_{0} e k} \sqrt{B_{V}}, \\
& \sigma_{B}=\sqrt{B_{\beta} \beta_{x}^{0}}, \quad \sigma_{R}=\sqrt{A_{D}-\sigma_{B}^{2}},
\end{aligned}
$$

where

$$
B_{\beta}=\sigma_{I}^{2}\left(\beta_{y}^{0}+0.25 L^{2} \gamma_{y}^{0}-L \alpha_{y}^{0}\right)^{-1} .
$$

Equation (11) calculates the coefficient $B_{\beta}$ from the results of the TDS voltage scan, Eq. (8). Otherwise, if we had measured the slice emittance $\epsilon_{n}$ independently, then we can use the more accurate estimation of $B_{\beta}$ through the relation $B_{\beta}=\epsilon_{n} / \gamma_{0}$. For example, we can estimate $B_{\beta}$ (or emittance $\epsilon_{n}$ ) by changing on $\beta_{x}$ at the OTR screen position and by keeping the dispersion $D$ constant and fitting the measured slice size $\sigma_{M}$ to the linear polynomial:

$$
\sigma_{M}^{2}=A_{\beta}+B_{\beta} \beta_{x} .
$$

We simulated with Eq. (1) the measurement of the beam size $\sigma_{M}$ for two scans as given by Eqs. (8)-(11). For the dispersion scan we used the values of $0.6,0.8,1.0$, and 1.2 metres. For the TDS voltage scan we used values 0.38 , $0.47,0.56,0.65$, and $0.75 \mathrm{MV}$. We used the same errors and the reconstruction algorithm as in the previous examples. The results of the reconstruction are shown in Fig. 3 and the
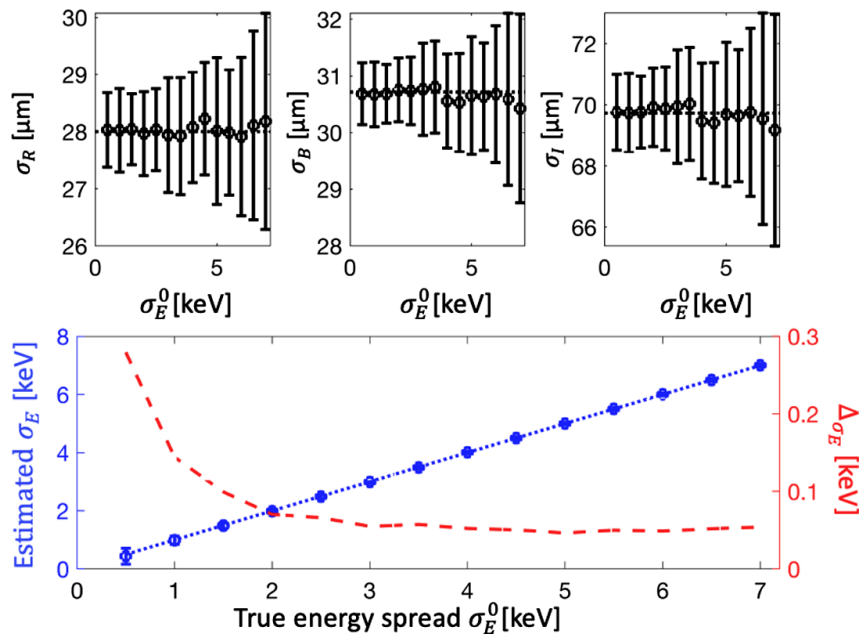

FIG. 3. Performance of the method based on dispersion scan. Circles shows reconstructed mean values. Error bars give the standard deviation error. The red dashed curve presents the rms error of the reconstruction. Dotted black curves show true values used for the simulation.

error of the reconstruction of energy spread is smaller than $0.1 \mathrm{keV}$ at the energy spread of $6 \mathrm{keV}$.

\section{Impact of systematic and random instrumental errors}

Finally, let us consider instrumental errors in the setup of the TDS voltage $V$ and the dispersion $D$ during the scans used in the dispersion scan method.

If the errors are systematic with the same sign then the error in reconstruction of the energy spread is inversely proportional to the error in the reference dispersion $D_{0}$ only. Indeed, we calculate the energy spread by Eq. (10)

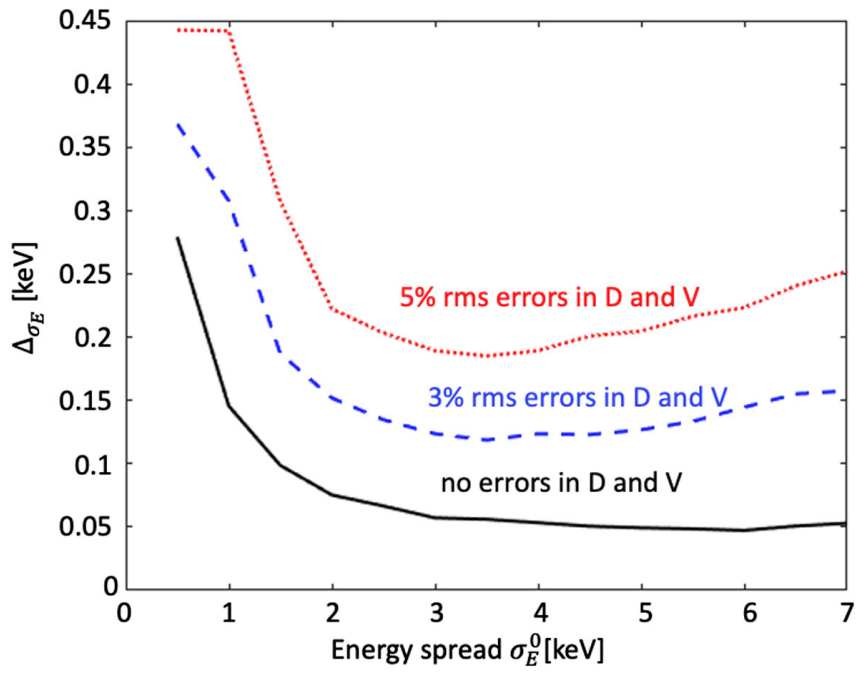

FIG. 4. The impact of instrumental errors in setup of voltage and dispersion on the reconstruction error from dispersion scan method. 


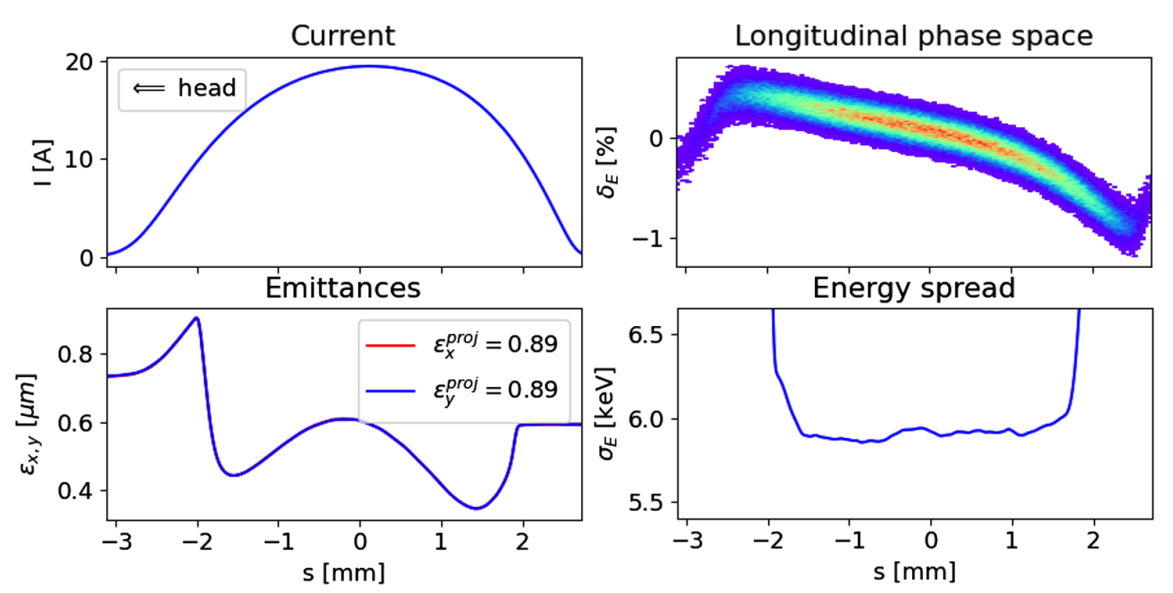

FIG. 5. Electron beam distribution after the gun used in the modeling.

and use only the constant terms $A_{D}$ and $A_{V}$. If we suggest that during the TDS voltage scan we set the voltage with the same negative error, for example at $10 \%$, then it has only impact the coefficient $B_{V}$ which in this case will be increased by factor $0.9^{-2}$, but the constant term $A_{V}$ is not changed. The same is true for the impact of the systematic error in the dispersion $D$ during the dispersion scan. Hence, only the systematic error in the reference dispersion $D_{0}$ affects the accuracy of reconstruction of the energy spread.

Next we analyze only random errors in the setup of voltage or dispersion. The results of the analysis are shown in Fig. 4. In the suggestion of rms error of $5 \%$ the rms reconstruction error remains below $0.3 \mathrm{keV}$ for the energy spread of $6 \mathrm{keV}$.

\section{MODELING OF THE EXPERIMENT WITH COLLECTIVE EFFECTS AND BEAM TRANSPORT}

The electron beam dynamics at the European XFEL accelerator have been recently discussed in $[8,9]$. In the latter work, an experimental validation of the collective effects modeling at the European XFEL injector was presented. Here we use the same approach from [9] to simulate the beam dynamics, namely (1) the dynamics of the electron beam in the gun was simulated using ASTRA code [10], (2) the beam tracking starting from $3.2 \mathrm{~m}$ from the gun cathode, was performed using the OCELOT code [11] with the space charge and the wakefield effects included, and (3) the coherent synchrotron radiation was omitted as it is negligible for this section.

In the measurements described in Sec. IV we have found that the uncorrelated energy spread is equal to approximately $5.9 \mathrm{keV}$. As it is discussed in Sec. V one of the possible reasons for such large energy spread could be intrabeam scattering (IBS) and the microbunching instability (MBI) in the lower energy part of the injector. The codes ASTRA and OCELOT do not model IBS. MBI was also not taken into account in the simulation of the experiment due to the relatively small number of particles (200k). In the simulation of the rf gun with ASTRA using a bunch charge of $250 \mathrm{pC}$ we obtain the energy spread of $0.6 \mathrm{keV}$. Hence we apply random generator at a distance of $3.2 \mathrm{~m}$ from the cathode to increase the energy spread artificially to $5.9 \mathrm{keV}$. The properties of the electron bunch after this procedure at position $z=3.2 \mathrm{~m}$ are shown in Fig. 5. Let us note here that the projected emittance at this distance from the cathode is relatively large. The emittance will reduce in the booster considerably according with the emittance compensation process [12].

\section{A. Magnetic lattice and its properties}

A special optics (shown in Fig. 6 and Table I) was developed to fulfil requirements of the experiments described above. The important properties of the optics are: (1) high dispersion at the screen position to maximize energy spread contribution to the beam size on the screen; (2) the high value of $R_{34}$ element of the transport matrix

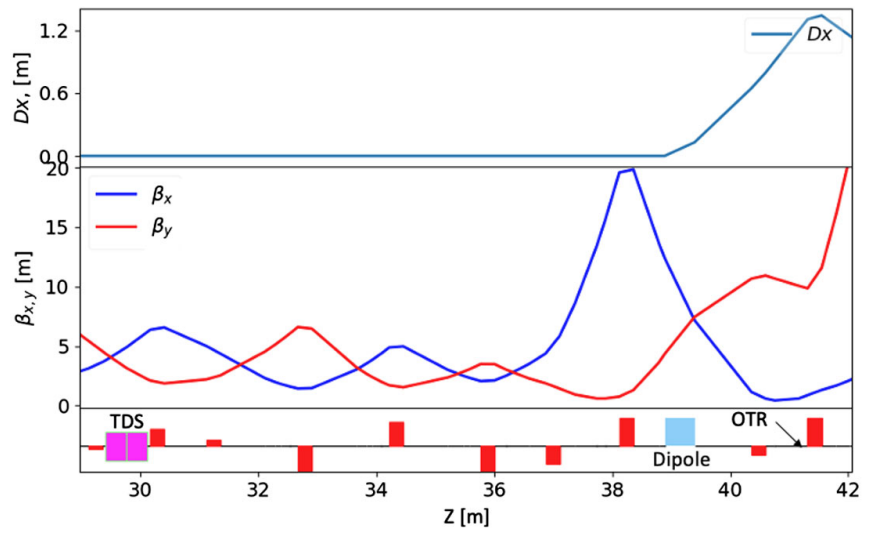

FIG. 6. Special optics for the slice energy spread measurement. Optics is shown from the matching point. Note, $\beta$-functions are calculated in linear approximation without any collective effects. 

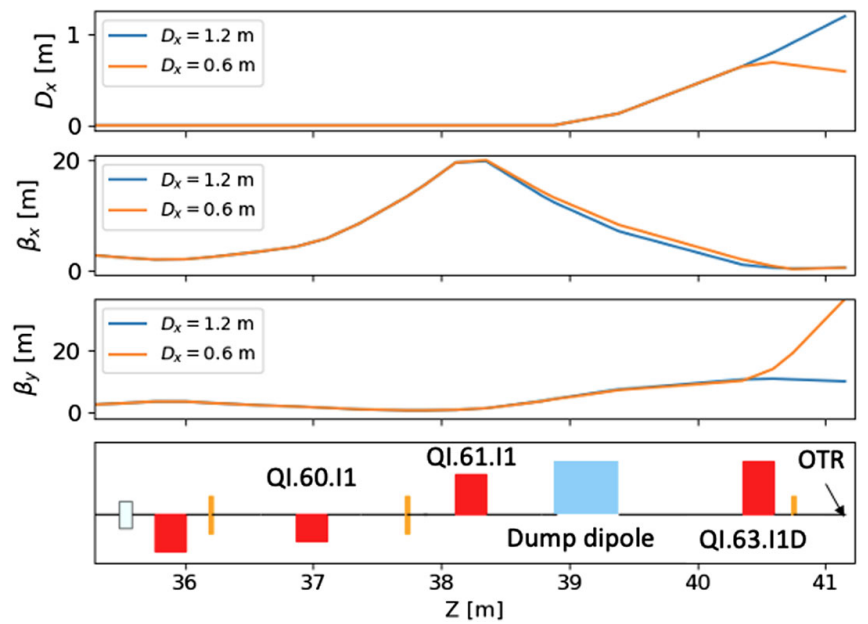

FIG. 7. Changes of the Twiss parameters in the dump section during dispersion scan.

between the TDS and the screen to minimize voltage of the TDS which in turn minimizes the induced energy spread; and (3) low sensitivity of $\beta_{x}$ in the screen position to the beam mismatch at the matching point before the TDS. To achieve this, we designed 8 different beam optics that met the first two criteria, and then chose an optics that had minimal changes in $\beta_{x}$ in the OTR position in response to the $\beta_{x}$ and $\alpha_{x}$ mismatch introduced before the TDS.

Another feature of this optics is the ability to vary the horizontal dispersion over a wide range of values without change in the horizontal $\beta$-function on the OTR screen. The main contribution for the dispersion change comes from the quadrupole QI.63.I1D while minor changes in the $\beta_{x^{-}}$ function are compensated by quadrupoles QI.60.I1 and QI.61.I1, as shown in Fig 7. The described feature is used to performed the dispersion scan.
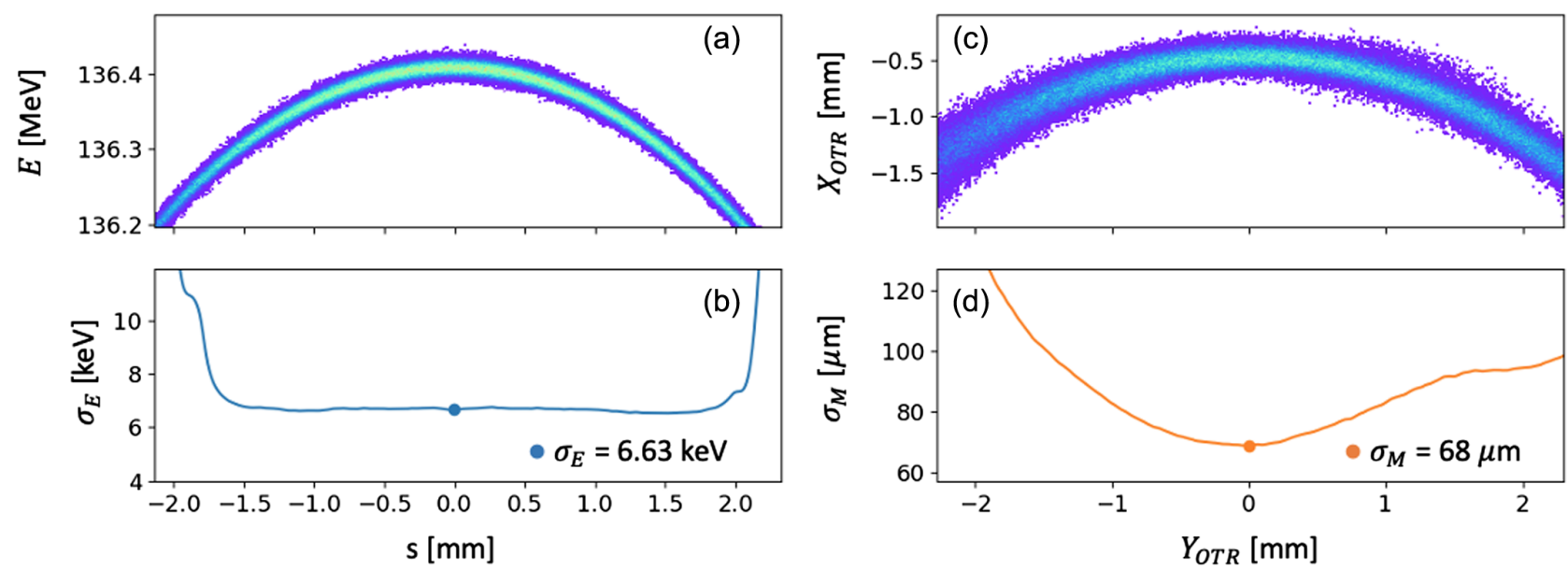

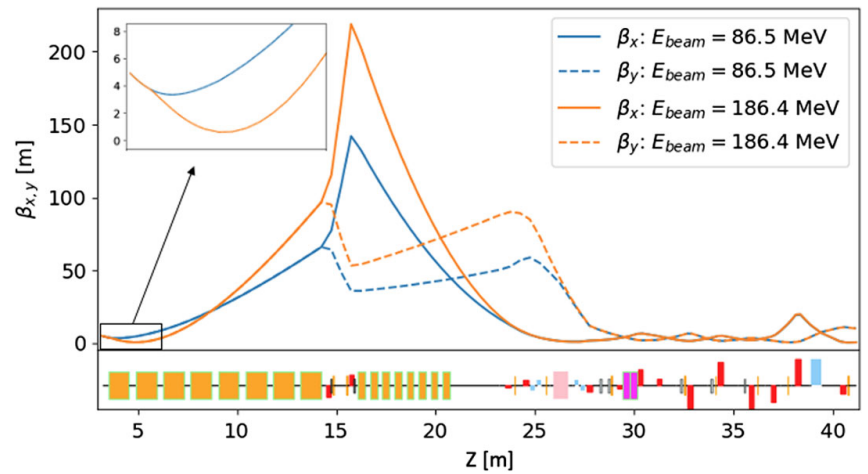

FIG. 9. $\quad \beta$-functions for lowest and highest energy while taking into account the space charge effect and rf focusing.

\section{B. Numerical modeling of the experiment based on the energy scan}

Following the measurement procedure described in the Sec. II A, we performed beam dynamics simulations changing the voltage of module A1 (see Fig. 1) from 80 to $180 \mathrm{MV}$ in step of $10 \mathrm{MV}$. The beam comes from the gun with an initial energy of $6.5 \mathrm{MeV}$.

The transport matrix from the TDS to the screen in the dump section has nonzero $R_{51}$ and $R_{52}$ elements. These elements create couplings between the horizontal and longitudinal planes, which leads to a broadening of the beam in the longitudinal direction, in case the beam has a nonzero horizontal emittance. In the presence of the correlated energy spread it will cause the slice widening on the OTR screen. This effect in simulations can be see in Fig. 8. On the left are two plots of the LPS (longitudinal phase space) beam distribution (a) and the slice energy spread (b) of the beam in front of the dump magnet, and on the right two plots are the image (c) and the horizontal slice

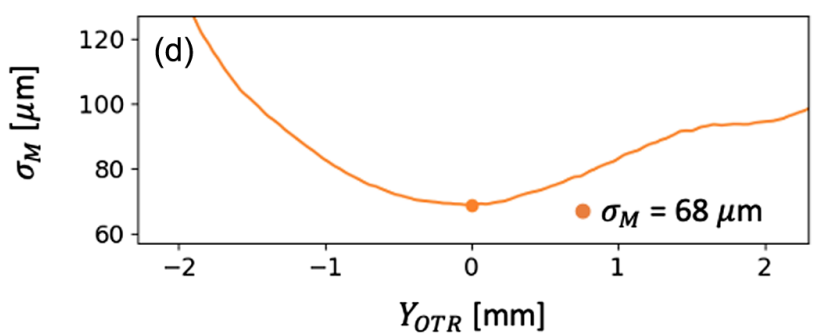

FIG. 8. Some details of longitudinal beam dynamics for the beam energy $136 \mathrm{MeV}$. (a) The longitudinal phase space (LPS) beam distribution in front of the dump magnet, (b) slice energy spread of the beam in front of dump magnet, (c) the beam image on the OTR screen, and (d) horizontal slice beam size on the OTR screen without effect of the screen resolution. 
TABLE II. The true and the reconstructed data from the beam dynamics simulations at the reference energy $E_{0}=130 \mathrm{MeV}$.

\begin{tabular}{lccccc}
\hline \hline Parameter & $\sigma_{E}$ & $\sigma_{I}$ & $\sigma_{B}$ & $\sigma_{R}$ & $\epsilon_{n}$ \\
\hline Units & $\mathrm{keV}$ & $\mu \mathrm{m}$ & $\mu \mathrm{m}$ & $\mu \mathrm{m}$ & $\mu \mathrm{m}$ \\
\hline True values & 5.90 & 80.3 & 35.4 & 28 & 0.53 \\
Energy scan method & 5.89 & & & 41 & \\
Dispersion scan method & 5.97 & 81.8 & 36.0 & 26.4 & 0.55 \\
\hline \hline
\end{tabular}

size (d) on the OTR screen of the same beam that was tracked through the dump section. To avoid the influence of this effect on the measurement, the beam slice of interest must have zero energy chirp. For completeness, it is worth noting that the element $R_{56}$ is small and its effect can be neglected.

Taking into account the effect described above, the third harmonic cavity AH1 was turned off. Measurement of the slice energy spread was carried out at the extremum of the mean slice energy. The horizontal Twiss parameters of the slice have been matched to the magnetic lattice before TDS. Since the rf-focusing effect is strong, the beam should be matched for all beam energies. At the Fig. 9 are shown $\beta$-functions of the central slice for highest and lowest energies. Twiss parameters were calculated from the beam transported in OCELOT with collective effects included.

In the simulations and in the experiment we have seen increase of the slice emittance by $30 \%$ at the highest voltage of rf module A1. It is due to very strong rf focusing and very small $\beta$-functions in module A 1 which, in turn, enhance the SC (space charge) effect. Additionally we think that IBS would change the energy spread during the energy scan as well, but this effect was omitted in the

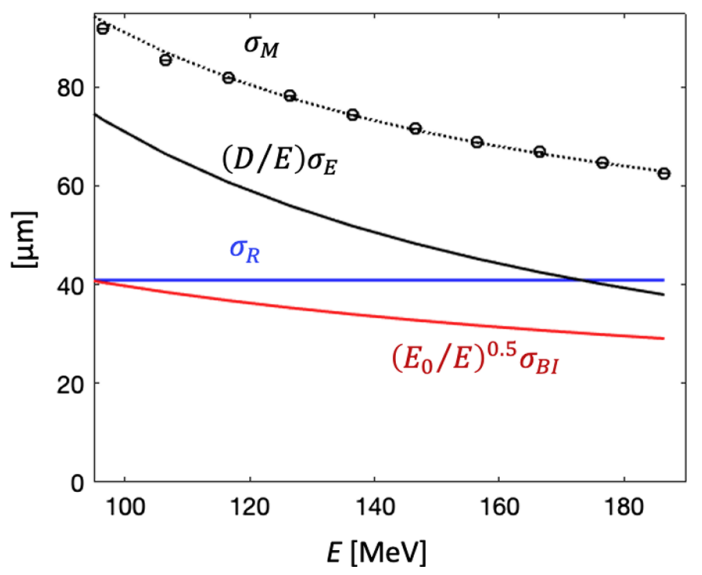

simulation. The true values used in the simulation are listed in the first row of Table II.

The results of the simulations are shown on Fig. 10 and in the second row of Table II. The left plot shows the results for the beam matched at each energy. The right plot shows the results for the beam matched only at the reference energy $E_{0}=130 \mathrm{MeV}$. The black circles show the slice width $\sigma_{M}$ from the simulations. The black dotted line at the left plot shows the results of the reconstruction with the method described in Sec. II A using Eq. (7). The other lines at this plot show the contribution of different terms of Eq. (7) as found from the reconstruction.

For the data shown in the right plot of Fig. 10 the reconstruction was impossible. The black dotted line shows the expected values calculated by Eq. (7) using the true data listed in the first row of Table II. The other lines at this plot show the contribution of different terms of Eq. (7) as found from the true values of Table II.

To summarize, in the setup of the European XFEL the slice matching procedure should be applied on each step. However, even with the beam matching, the reconstruction could be inaccurate due to changes in the slice emittance and the energy spread at different energies.

\section{Numerical modeling of the experiment based on dispersion scan}

Another method for the slice energy spread measurement proposed in the Sec. II B uses the variation of the dispersion instead of the energy. To test the viability of the method, we carried out numerical experiments similar to those described above with two scans: dispersion scan and TDS voltage scan.

The simulations have been done at the reference beam energy of $130 \mathrm{MeV}$ for two different uncorrelated energy

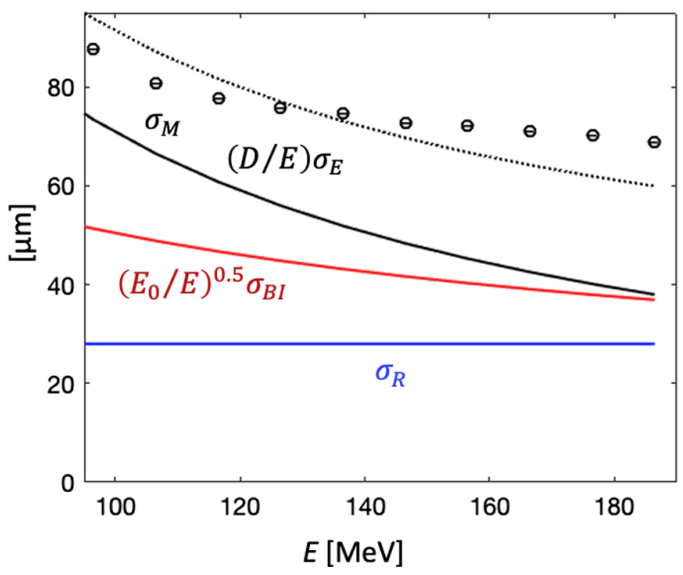

FIG. 10. The left plot shows the results for the beam matched at each energy. The right plot shows the results for the beam matched only at the reference energy $E_{0}=130 \mathrm{MeV}$. The black circles show the slice width $\sigma_{M}$ from the simulations. The black dotted line in the left plot shows the results of the reconstruction with Eq. (7). The other lines in this plot show contribution of different terms. For the data shown in the right plot the reconstruction is impossible. The lines show the expected values calculated using the true data of Table II. 

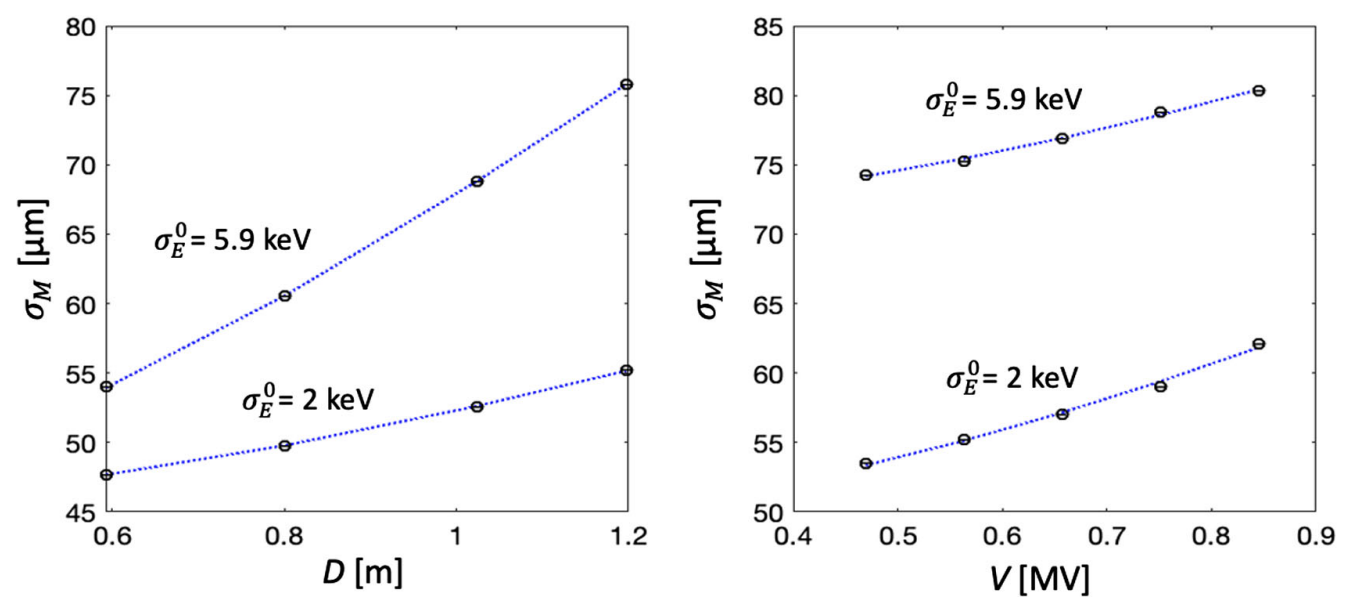

FIG. 11. Beam size $\sigma_{M}$ (black circles) from the dispersion scan and the TDS voltage scans as obtained from beam dynamics simulations. The blue dotted lines presents the curves of the numerical fit with Eq. (9) and Eq. (8).

spreads: $5.9 \mathrm{keV}$ and $2 \mathrm{keV}$. The dispersion scan was performed first on the OTR screen with the values of 0.6, $0.8,1.0$, and $1.2 \mathrm{~m}$. The TDS voltage was kept constant at $0.61 \mathrm{MV}$. The second scan was performed with TDS voltages of $0.47,0.56,0.65,0.75,0.84 \mathrm{MV}$. The dispersion was kept constant at $1.2 \mathrm{~m}$.

The results of the modeling are shown in Fig. 11. The black circles show the central slice width $\sigma_{M}$ obtained from the beam dynamics simulations. The blue dotted lines presents the curves reconstructed using the method described in Sec. II B.

Using the reconstruction procedure described in Sec. II B for the true energy spread of $5.9 \mathrm{keV}$ we got the reconstructed values listed in the last row of Table II. We see that all values are reconstructed with high accuracy. For the true energy spread of $2 \mathrm{keV}$ the reconstructed energy spread is $2.13 \mathrm{keV}$. Contrary to the first method based on energy scan in the second method the slice emittance does not change during the dispersion and the TDS voltage scans.

\section{MEASUREMENTS}

In the analysis of the images obtained in the experiment we have followed the same procedure as in the simulations. At each point we took 30 images and for each of them we calculated the mean slice energy and the slice width. The image processing was performed with a fixed bin size (slice length) in pixels, which corresponds to 0.2 ps for a TDS voltage of $0.61 \mathrm{MV}$, and the slice width was found by fitting it with a Gaussian form. Then the slice width at the extremum of the mean slice energy curve has been taken as $\sigma_{M}$.

From the measurements we estimate that the standard deviation error in $\sigma_{M}$ is below $1.5 \%$. Hence the error in the mean value from the 30 measurement is below $0.3 \%$.

Due to substantial difficulties with the energy scan method in the experiment we change the order of consideration and consider the dispersion scan method at the beginning.

\section{A. Results obtained with the dispersion scan}

The measurements presented in this section have been conducted at the constant electron beam energy of $E_{0}=130 \mathrm{MeV}$. The parameters of the gun have been optimized to have a small normalized slice emittance of $\epsilon_{n} \approx 0.4 \mu \mathrm{m}$.

At the first step of the energy scan method we conducted the measurements at constant dispersion $D_{0}=1.181 \mathrm{~m}$ with different TDS voltages $V$. The TDS voltages and the measured values of the slice width $\sigma_{M}$ together with the errors are listed in the Table III.

At the second step we conducted the measurements at a constant TDS voltage of $V_{0}=0.61 \mathrm{MV}$ with different dispersion values $D$. The dispersion values and the measured values of the slice width $\sigma_{M}$ together with the errors are listed in the Table IV.

Figure 12 shows the curves measured in the core of the electron bunch. At the position of the OTR screen the electron bunch has rms length of 4 ps with the TDS voltage $0.61 \mathrm{MV}$. Figures (a) and (b) show the vertical position of

TABLE III. The beam sizes measured during the TDS scan, $D=1.181 \mathrm{~m}$.

\begin{tabular}{lcccccc}
\hline \hline$V$ & MV & 0.375 & 0.469 & 0.563 & 0.657 & 0.751 \\
$\sigma_{M}$ & $\mu \mathrm{m}$ & $69.87 \pm 0.12$ & $70.64 \pm 0.10$ & $71.86 \pm 0.13$ & $72.85 \pm 0.17$ & $74.12 \pm 0.14$ \\
\hline \hline
\end{tabular}


TABLE IV. The beam sizes measured during the dispersion scan, $V=0.61 \mathrm{MV}$.

\begin{tabular}{lccccc}
\hline \hline$D$ & $\mathrm{~m}$ & 0.578 & 0.789 & 1.006 & 1.181 \\
$\sigma_{M}$ & $\mu \mathrm{m}$ & $50.62 \pm 0.08$ & $57.49 \pm 0.09$ & $65.43 \pm 0.1$ & $72.05 \pm 0.1$ \\
\hline \hline
\end{tabular}

the slice $y$ on the screen. Figures (c) and (d) show the slice width on the screen. The gray dotted line defines the position of the reference slice. It is the extremum of the curves shown in plots (a) and (b). As one can see, the reference slice do not have the minimal width but its position well defined by the extremum of the mean slice position curve. We observed the same behavior in the simulations, the reason being that the slice emittance is not constant along the beam. Note, (1) Fig. 12(b) contains 5 curves obtained with the different TDS streak factors, (2) the TDS scan images were processed with the bin size (slice length) varied from $0.33 \mathrm{ps}$ at the lowest TDS voltage to $0.16 \mathrm{ps}$ at the highest.

The measured values from Table III, IV correspond to the coordinate $t=0 \mathrm{ps}$ in Fig. 12. They are plotted in Fig. 13 using black circles with error bars. The blue dotted lines are obtained by the numerical fit to Eq. (9) (left plot) and Eq. (8) (right plot).

The coefficients of Eq. (8) and Eq. (9) obtained from the scans are listed in Table V. Shown in the same table are the physical values of interest with the estimated errors. They are obtained using Eqs. (10) and (11). The errors
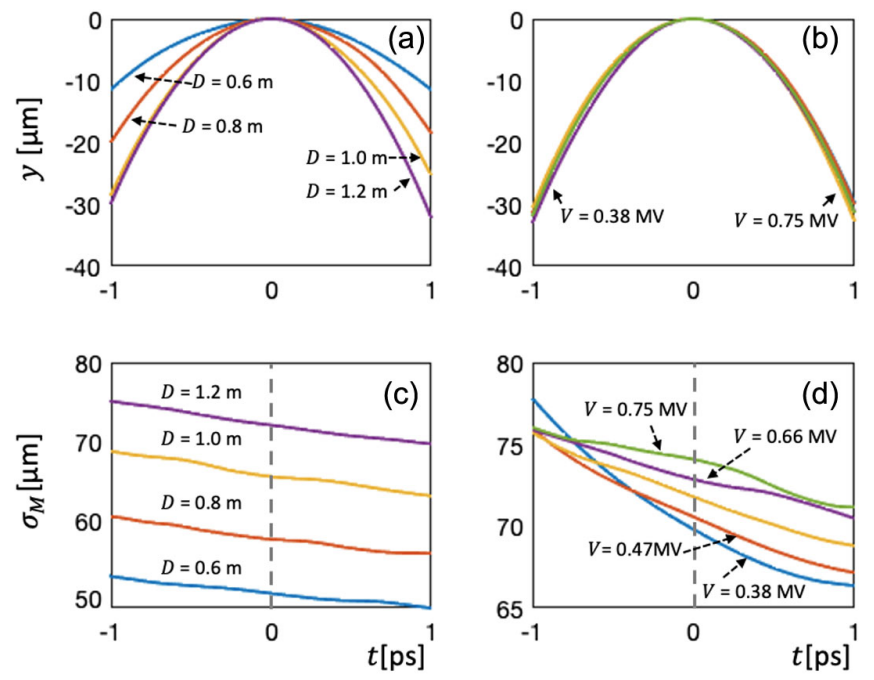

FIG. 12. Measured curves with the dispersion scan method. Mean vertical position of the slices $y$ on the screen along the bunch for different dispersion values are shown in (a). Mean vertical position of the slices on the screen along the bunch for different TDS voltages are shown in (b). Vertical size of the slices on the screen along the bunch for different dispersion values are shown in (c). Vertical size of the slices on the screen along the bunch for different TDS voltages are shown in (d). The gray dotted lines present the position of the reference slice.
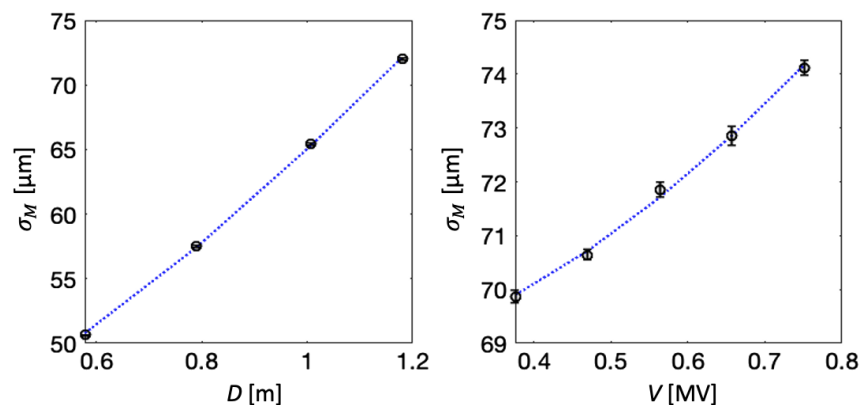

FIG. 13. The black circles with error bars at the left plot show the measured slice width $\sigma_{M}$ for different dispersion values $D$. The black circles with error bars at the right plot show the measured slice width $\sigma_{M}$ for different values of the TDS voltage $V$. The blue dotted lines are obtained by the numerical fit to Eq. (8) and Eq. (9).

are estimated by the numerical experiment described in Sec. II A.

If we take into account that the estimated instrumental errors in the setup of the TDS voltage and dispersion are smaller than $2 \%$ then we can state that the uncorrelated energy spread in the core of the beam is equal to $(5.9 \pm 0.1) \mathrm{keV}$.

Note that the emittance estimation agrees well with the independent method of the measurement of the beam emittance (see Fig. 14). Finally the estimated screen resolution $\sigma_{R}$ agrees with the numbers published in [4,6].

\section{B. Results obtained with the energy scan}

We have done the energy scans with dispersion values of 0.6 and 1.2 metres, with results of these scans shown in Fig. 15. We were not able to do the reconstruction from the measured data and we simply compared the measurements with the expected values calculated from the results of the previous method presented in the Table V.

Taking into account the issues with the beam matching and nonconstant slice emittance (see Sec. III B) we think that there is no contradiction between the data.

It is shown in Sec. III B that the energy scan method could be used but requires stringent control of the shape of the longitudinal phase space and very accurate matching of the beam to the optics before the TDS. Unfortunately, after two very time-consuming experiments, we were still unable to show this.

\section{Validation of the experimental results}

In this section we consider several arguments to confirm the accuracy of the obtained data.

The energy spread estimation based on Eq. (10) uses only coefficient $A_{V}$ and $A_{D}$, but there is another equation

$$
\sigma_{E}=\frac{E_{0}}{D_{0}} \sqrt{D_{0}^{2} B_{D}-V_{0}^{2} B_{V}},
$$


TABLE V. The reconstructed data from the measurements with the dispersion scan method.

\begin{tabular}{lcccccccc}
\hline \hline$A_{V}$ & $B_{V}$ & $A_{D}$ & $B_{D}$ & $\sigma_{E}$ & $\sigma_{I}$ & $\sigma_{B}$ & $\sigma_{R}$ & $\epsilon_{n}$ \\
\hline$m^{2}$ & $m^{2} / M V^{2}$ & $m^{2}$ & & $\mathrm{keV}$ & $\mu \mathrm{m}$ & $\mu \mathrm{m}$ & $\mu \mathrm{m}$ & $\mu \mathrm{m}$ \\
\hline $4.68 \mathrm{e}-9$ & $1.45 \mathrm{e}-9$ & $1.75 \mathrm{e}-9$ & $2.48 \mathrm{e}-9$ & $5.948 \pm 0.06$ & $71.4 \pm 3$ & $31.4 \pm 1.3$ & $27.6 \pm 1.5$ & $0.42 \pm 0.02$ \\
\hline \hline
\end{tabular}

based on two other coefficients, $B_{D}$ and $B_{V}$, from the numerical fits. From Eq. (13) we ascertain that the energy spread is equal to $5.946 \mathrm{keV}$, which agrees with the previous estimation (see Table V) with an accuracy $0.03 \%$.

In order to check the estimation of the emittance $\epsilon_{n}$ we have done an independent measurement of the slice emittance with the standard tools [13] used by operators of the facility. The results of independent measurement of the slice emittance are shown in Fig. 14 and the emittance of the central slice (slice index 0 ) agrees with the value listed in Table V.
We had the additional possibility to do the measurement of the slice energy spread with the laser heater tuned for maximal SASE radiation energy. We have found that the energy spread in the electron bunch was $7.5 \pm 0.1 \mathrm{keV}$.

In theoretical studies of microbunching carried out by our colleague M. Dohlus (see, for example, [15]) the optimal energy spread after the laser heater for microbunching suppression is nearly $8 \mathrm{keV}$. This value agrees reasonably well with the measured one.
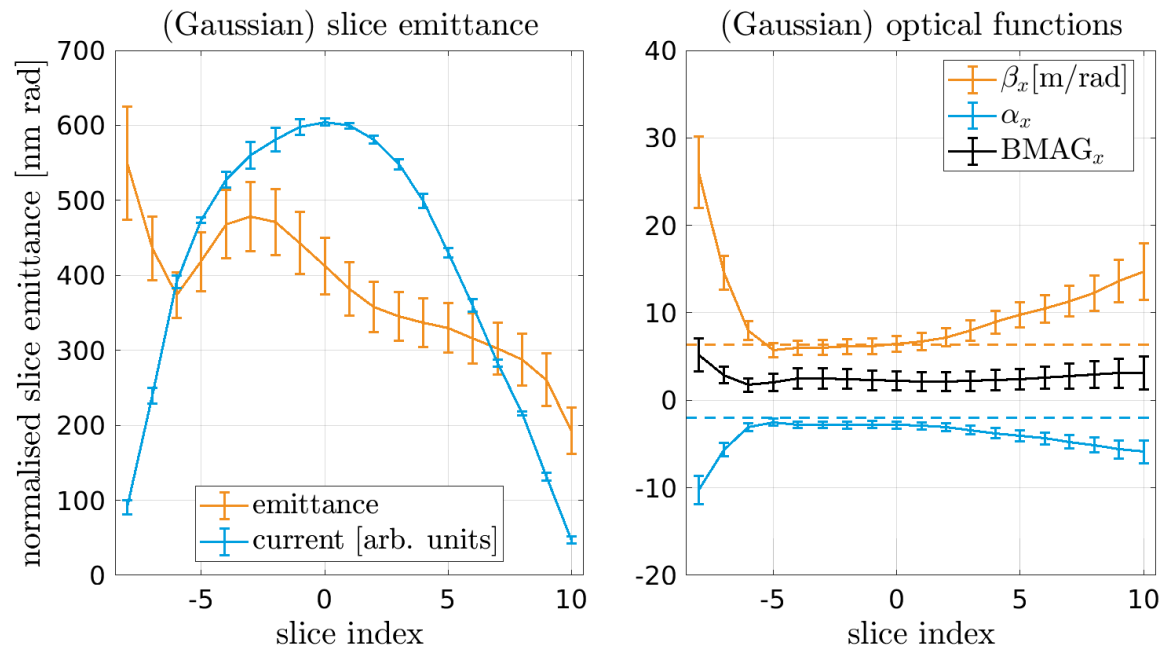

FIG. 14. The slice emittance along the bunch measured by the standard method used at the facility [13]. Black line in the right plot corresponds to the BMAG parameter [14].
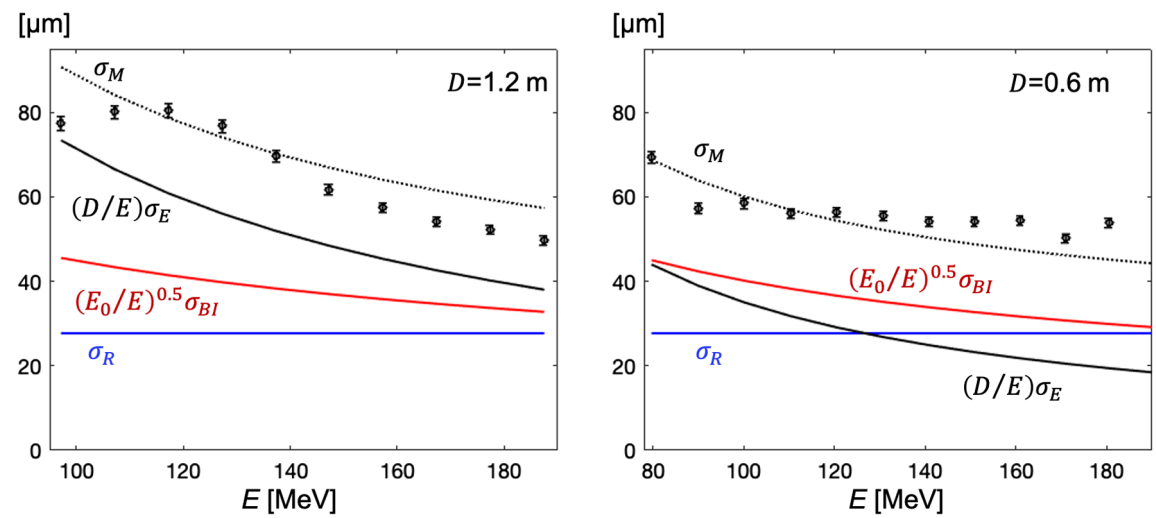

FIG. 15. Comparison of the measurements of the energy scan (dots with error bars) with the values calculated from the data of the dispersion scan. 


\section{DISCUSSION}

The theoretical calculations with different numerical models predict the uncorrelated energy spread to be below $1 \mathrm{keV}$. The discrepancy between the theoretical estimations and the measurements could be caused by neglecting the full physics when using the simplified numerical models. For example, it could be that the emission process from the cathode should be simulated differently. Additionally we do not take into account the intrabeam scattering and wakefields in the rf gun cavity. Finally, the number of macroparticles used in the simulations does not allow to take into account the microbunching instability effect during the transport from the gun to the OTR screen.

It was shown in $[16,17]$ that the intrabeam scattering in the injector section increases the energy spread considerably and has to be taken into account. For example, a simple estimation of the induced energy spread due to IBS from [17] is

$$
\sigma_{E}^{\mathrm{IBS}}=\sqrt{\frac{2 r_{e}^{2} N_{b}}{\epsilon_{n}} \int \frac{d s}{\sigma_{x} \sigma_{z}}},
$$

where $r_{e}$ is the electron radius, $N_{b}$ is the number of electrons, $\sigma_{z}$ is the rms length of the bunch, $\sigma_{x}$ is the transverse rms size and the integration is done along the bunch path $s$. If we use this equation with the parameters used in the paper we obtain the energy spread introduced during the beam transport from the gun to OTR to be about $2 \mathrm{keV}$. Hence, it is a considerable effect and should be taken into account in the simulations. We are now evaluating different models of IBS to be included in the beam dynamics codes.

The energy spread from the rf gun measured at the European XFEL for a bunch charge of $250 \mathrm{pC}$ is $5.9 \pm 0.1 \mathrm{keV}$. This number is approximately 3 times lower then the energy spread of $14.8 \pm 0.6 \mathrm{keV}$ reported recently by SwissFEL for the bunch charge of $200 \mathrm{pC}$ [4]. Both guns use cesium telluride cathodes and the larger difference between these results requires additional effort to understand. One possible reason could be that in the case of SwissFEL, the screen is located farther from the cathode than in our case, and IBS and other collective effects contribute more.

\section{SUMMARY}

We have described two methods for measurement of the slice energy spread of an electron bunch. With the beam dynamics simulations we have identified substantial difficulties of the first method based on energy scan: we need match the beam and the slice emittance changes. The difficulties are confirmed in the real experiment.

We have shown with the beam dynamics simulations and the measurements that the second method based on dispersion scan at the constant beam energy shows high accuracy and easy to conduct.

At the same time the measured slice energy spread of $5.9 \pm 0.1 \mathrm{keV}$ is several times higher than theoretically estimated and it requires additional theoretical research to clarify.

\section{ACKNOWLEDGMENTS}

The authors thank M. Dohlus, M. Krasilnikov, and S. Walker for helpful discussions and corrections. We thank members of the European XFEL team for providing help and conditions to carry out the measurements.

[1] Z. Huang and K.-J. Kim, Formulas for coherent synchrotron radiation microbunching in a bunch compressor chicane, Phys. Rev. ST Accel. Beams 5, 074401 (2002).

[2] E.Saldin, E.Schneidmiller, and M. Yurkov, Klystron instability of a relativistic electron beam in a bunch compressor, Nucl. Instrum. Methods Phys. Res., Sect. A 490, 1 (2002).

[3] J. Qiang, Y. Ding, P. Emma, Z. Huang, D. Ratner, T. O. Raubenheimer, M. Venturini, and F. Zhou, Start-to-end simulation of the shot-noise driven microbunching instability experiment at the Linac Coherent Light Source, Phys. Rev. Accel. Beams 20, 054402 (2017).

[4] E. Prat, P. Dijkstal, E. Ferrari, A. Malyzhenkov, and S. Reiche, High resolution dispersion-based measurement of the electron beam energy spread, Phys. Rev. Accel. Beams 23, 090701 (2020).

[5] M. Krasilnikov, dE-measurement program, in Proceedings at DESY beam dynamics meeting, Zeuthen, February 2016.

[6] C. Wiebers, M. Holz, G. Kube, D. Noelle, G. Priebe, and H.-Ch. Schroeder, Scintillating screen monitors for transverse electron beam profile diagnostics at the European XFEL, in Proceedings of IBIC2013, Oxford, UK (JACoW, Geneva, 2013), WEPF03.

[7] J. C. Lagarias, J. A. Reeds, M. H. Wright, and P. E. Wright, Convergence properties of the Nelder-Mead simplex method in low dimensions, SIAM J. Optimiz. 9, 112 (1998).

[8] I. Zagorodnov, M. Dohlus, and S. Tomin, Accelerator beam dynamics at the European x-ray free electron laser, Phys. Rev. Accel. Beams 22, 024401 (2019).

[9] I. Zagorodnov, S. Tomin, Y. Chen, and F. Brinker, Experimental validation of collective effects modeling at injector section of x-ray free-electron laser, Nucl. Instrum. Methods Phys. Res., Sect. A 995, 165111 (2021).

[10] K. Floettmann, ASTRA: A Space Charge Tracking Algorithm, DESY, 2017.

[11] S. Tomin, I.Agapov, M. Dohlus, and I. Zagorodnov, Ocelot as a framework for beam dynamics simulations of x-ray sources, in Proceedings of International Particle Accelerator Conference, Copenhagen, Denmark, 2017 (JACoW, Geneva, 2017), WEPAB031.

[12] B. E. Carlsten, New photoelectric injector design for the Los Alamos National Laboratory XUV FEL accelerator, 
Nucl. Instrum. Methods Phys. Res., Sect. A 285, 313 (1989).

[13] M. Scholz and B. Beutner, Electron beam phase space tomography at the European XFEL injector, in Proceedings of Iinternational Particle Accelerator Conference, Copenhagen, Denmark, 2017 (JACoW, Geneva, 2017), MOPAB047.

[14] M. Sands, A Beta mismatch parameter, SLAC Internal report, Report No. SLAC-AP-085, 1991.
[15] M. Dohlus, Microbunching in the European XFEL, in Proceeding at DESY beam dynamics meeting, DESY, May 2020.

[16] S. Di Mitri et al., Experimental evidence of intrabeam scattering in a free-electron laser driver, New J. Phys. 22, 083053 (2020).

[17] Z. Huang, Intrabeam Scattering in an X-ray FEL Driver, SLAC Report No. SLAC-TN-05-026, 2002. 